\title{
Preoperative Planning for Renal Cell Carcinoma - Benefits of 64-Slice CT Imaging
}

\author{
Manjiri Dighe, Thomas Takayama, William H. Bush Jr
}

Department of Radiology (MD, WHB), and Department of Surgery (TT), University of Washington Medical Center, Seattle, Washington, USA

\begin{abstract}
Surgery is the primary form of treatment in localized renal cell carcinoma. Adrenal-sparing nephrectomy, laparoscopic nephrectomy and nephron-sparing partial nephrectomy are growing trends for more limited surgical resection. Accurate preoperative imaging is essential for planning the surgical approach. Multislice CT and MR are regarded as the most efficient modalities for imaging renal neoplasms. Development of faster CT systems like 64-slice CT with improved resolution and capability to achieve isotropic reformats have significantly enhanced the role of CT in imaging of renal neoplasms. This review article describes the present state, technique and benefits of 64-slice CT scanning in preoperative planning for RCC.
\end{abstract}

Key words: renal cell carcinoma; imaging, three-dimensional; surgery

Int Braz. J Urol. 2007; 33: 305-12

\section{INTRODUCTION}

Renal cell carcinoma (RCC) is the fifth most common cancer in adults and accounts for $85 \%$ of all malignant tumors of the kidney (1). It represents $3 \%$ of all male and $2 \%$ of female cancers. The incidence of RCC is 6.0 - 8.0 per 100,000 of the population (2). Surgery is the primary form of treatment in localized renal cell carcinoma. In addition, adrenal-sparing nephrectomy, laparoscopic nephrectomy and nephronsparing partial nephrectomy are growing trends for more limited surgical resection (3). The aims of preoperative imaging are to characterize the lesion, identify accessory vessels, define adjacent organ extension, identify lymph nodal or visceral metastases, reliably predict the presence and extent of any thrombus in the renal vein, vena cava and heart and evaluate the other kidney.

Multislice CT and MR are regarded as the most effective modalities for imaging renal neoplasms. Development of faster CT systems like the 64-slice CT scanner with improved resolution and capability to achieve isotropic reformats have significantly enhanced the role of CT in preoperative imaging of renal neoplasms.

\section{IMAGING TECHNIQUE AND PROTOCOLS WITH 64 SLICE MULTIDETECTOR TECHNOLOGY}

Helical CT has dramatically refined the diagnostic evaluation of renal pathologic conditions by al- 
lowing image acquisition through the entire kidney during various phases of contrast enhancement after the administration of a single bolus of intravenous contrast material (4-7). Multi-detector 64-slice CT technology takes advantage of significantly reduced image acquisition time for multi-phasic, thin-slice image acquisitions of the kidneys in a single breath-hold with superior contrast bolus utilization.

A precontrast phase provides a baseline from which to measure the enhancement within the lesion after the administration of intravenous contrast material. Calcifications are also seen in 30\% of cases of RCC. The enhancement characteristic is important in distinguishing hyperdense cysts from solid tumors (Figures-1A and 1B). Because most renal cell carcinomas have a rich vascular supply, they enhance significantly after administration of intravascular contrast material. Enhancement values of more than 15-20 HU are considered suspicious for malignancy (8); more variation in enhancement is found with small masses due to the dense opacification of adjacent normal parenchyma.

Generally, dual phase imaging with a nephrographic phase and a delayed phase is used for evaluating suspected renal neoplasms and depicting perinephric extension and vascular anatomy. For dual phase imaging, $150 \mathrm{ml}$ of non-ionic lower osmolality contrast media $\left(320 \mathrm{mg} \mathrm{ml}^{-1}\right)$ is injected at $3 \mathrm{cc} / \mathrm{sec}$ and images are acquired following delays of approximately 90 seconds and 6 minutes for the nephrographic and pyelographic phases, respectively. In some institutions, an initial scan is also performed in the corticomedullary phase. In the corticomedullary phase, the contrast material is primarily in the cortical capillaries, peritubular spaces, and cortical tubular lamina and has not yet filtered through the more distal renal tubules (9). In the nephrographic phase, the contrast material filters through the glomeruli into the loops of Henle and collecting tubules (9). The renal parenchyma enhances homogenously during this phase, due to tubular accumulation. Renal tumors enhance less than the surrounding parenchyma in this phase, because they lack normal glomerular-tubular function and hence this phase is most valuable for detecting renal masses and characterizing indeterminate lesions (10). The contrast reaches the collecting system during the delayed or the excretory phase and, as a result, the attenuation of the nephrogram progressively decreases (Figure-1C). Macari \& Bosniak (11) have suggested that measurement of the washout of contrast material from a lesion at 15 minutes allows differentiation between hyperdense cysts and renal neoplasms. In their study, there was no change in the attenuation of high-density cysts between the initial contrast-enhanced CT scan and the 15-minutedelayed images. In comparison, all lesions that proved to be neoplasms at surgery or follow-up studies showed a decrease in attenuation or "de-enhancement" of at least $15 \mathrm{HU}$ at delayed CT, which was attributed to the washout of contrast material from the vascular bed of the tumor (11).

Contiguous thin sections are obtained using protocols dependent on machine manufacturer to enable overlapping reconstruction. At our institution, multiphase CT scans are obtained on GE Lightspeed $\mathrm{VCT}^{\mathrm{TM}} 64$ slice scanner at $0.625 \mathrm{~mm}$ thickness at a pitch of 1.375:1. These slices are reconstructed at 2.5 $\mathrm{mm}$ thickness in the axial plane and coronal reformats are obtained at $5 \mathrm{~mm}$ intervals. Sagittal reformats and $3 \mathrm{D}$ reconstruction are performed on a case specific basis. Cardiac gated studies are performed for patients with IVC extension (see Figure-4). Multiphase CT of the abdomen is performed from top of the liver to $1 \mathrm{~cm}$ below the lowest kidney. A 15 minute scanogram is performed at the end of the exam to assess the ureters. The protocol used at our institution is illustrated in Table-1.

With the rapid acquisition of multiple simultaneous slices and thin-section reformatted coronal and/ or sagittal images, the 64-slice CT scanner provides excellent detail in demonstrating local extension to the bowel and retroperitoneum (see Figures-2, 3, 5, 6 and 7). Multiplanar three-dimensional reconstruction techniques including volume rendering, maximum intensity projection and shaded surface display provide comprehensive information about the relationships and possible involvement of vascular structures in renal neoplasms.

\section{TECHNICAL CONSIDERATIONS}

Standard oral contrast media for pre-operative assessment of renal neoplasm and vasculature is 

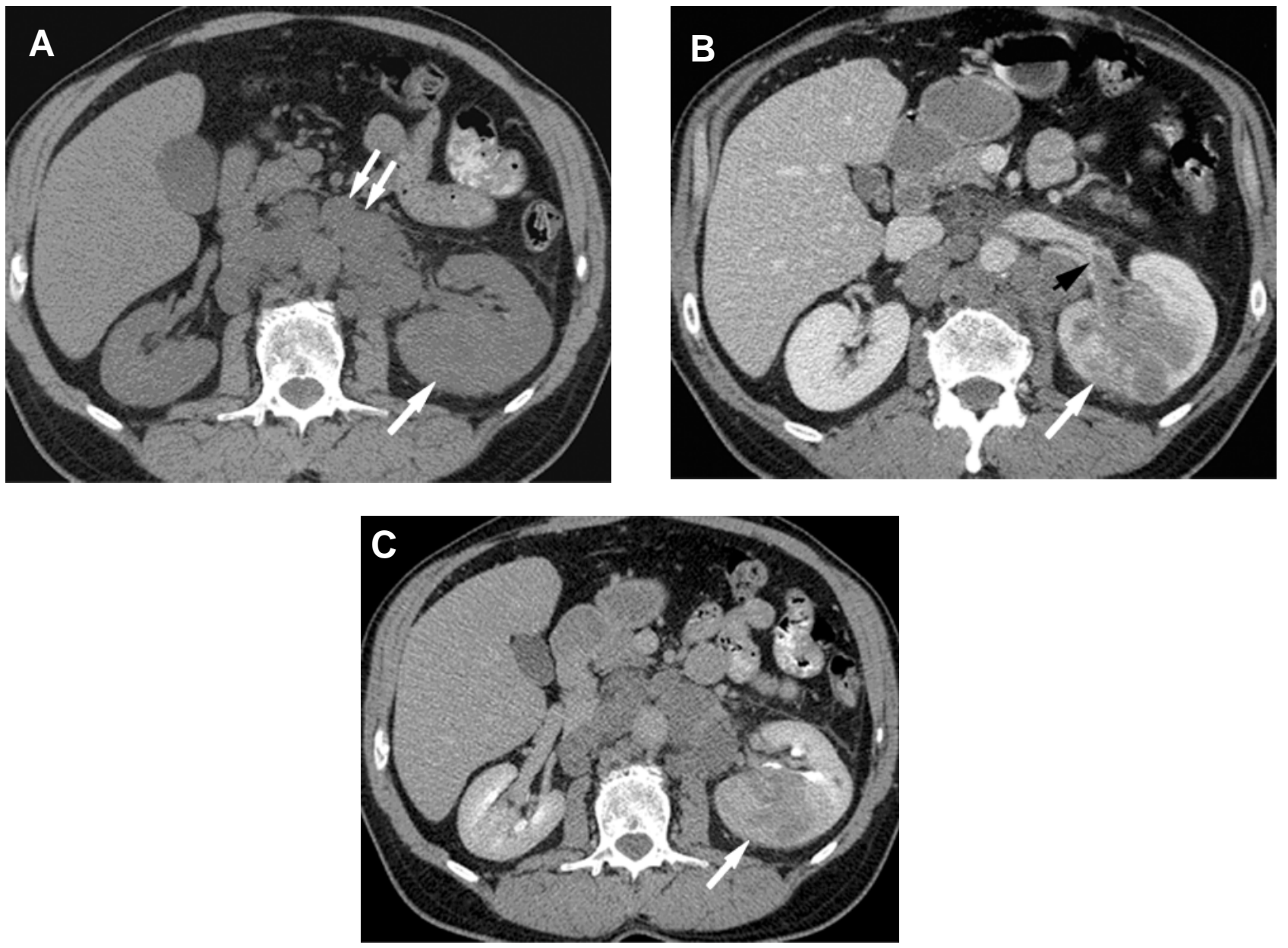

\begin{tabular}{cccccc} 
D & \multicolumn{5}{c}{ Dose Report } \\
Series & Type & $\begin{array}{c}\text { Scan Range } \\
(\mathrm{mm})\end{array}$ & $\begin{array}{c}\text { CTDlvol } \\
(\mathrm{mGy})\end{array}$ & $\begin{array}{c}\text { DLP } \\
(\mathrm{mGy}-\mathrm{cm})\end{array}$ & $\begin{array}{c}\text { Phantom } \\
\mathrm{cm}\end{array}$ \\
1 & Scout & & & & \\
2 & Helical & $170.250-1325.250$ & 7.57 & 242.00 & Body 32 \\
3 & Helical & $1147.000-1312.000$ & 5.08 & 116.62 & Body 32 \\
3 & Helical & $1147.000-1312.000$ & 5.08 & 116.62 & Body 32 \\
4 & Scout & \multicolumn{2}{c}{ Total Exam DLP: } & 475.24 &
\end{tabular}

Figure 1 - Axial CT scan of the abdomen showing renal cell carcinoma. A) The tumor is involving the left kidney (arrow) with nodal metastases in the retroperitoneum (double arrow). Note the noncontrast isodense appearance of the mass compared to the rest of the kidney. B) This image illustrates the enhancement in the mass on contrast administration where it increased in enhancement from $20 \mathrm{HU}$ to $110 \mathrm{HU}$. Note the small thrombus in the proximal left renal vein (black arrowhead). C) Delayed image through the kidney showing the close proximity of the mass to the renal collecting system. D) A typical dose report generated automatically by the scanner for a patient. Dose given is calculated for each scan (noncontrast [series 2], nephrographic [series 3a] and delayed phase [series 3b] with the total dose of $475.24 \mathrm{mGy}$-cm for this patient). 
Table 1 - Protocol used in our institution.

\begin{tabular}{ll}
\hline IV contrast & $150 \mathrm{cc}$ Optiray ${ }^{\top \mathrm{M}} 320,3 \mathrm{cc} / \mathrm{sec}$ \\
Precontrast & Diaphragm to iliac crest \\
90 sec delay & $1 \mathrm{~cm}$ above the top of the kidney to $1 \mathrm{~cm}$ below the lowest kidney \\
6 min delay & $1 \mathrm{~cm}$ above the top of the kidney to $1 \mathrm{~cm}$ below the lowest kidney \\
15 min delay & AP scout view from diaphragm to base of bladder \\
Pitch & $1.375: 1$ \\
Slice thickness & $0.625 \mathrm{~mm}$ \\
$\mathrm{kVp} /$ helical rotation & $120-140 / 0.4-0.8 \mathrm{sec}$ \\
Recon 1 & $2.5 \mathrm{~mm}$ standard \\
Recon 2 & Coronal $5 \mathrm{~mm}$ \\
\hline
\end{tabular}

unnecessary and may, in fact, obscure intravascular contrast. The radiation dose in our CT scanners is regulated using the Smart $\mathrm{mA}^{\mathrm{TM}}$ feature, in which the mAs used is regulated according to the amount of tissue (bone/soft tissue) that has to be traversed in $\mathrm{x}$, $y$ and $z$ axes. For example, the mAs used in the midabdomen is less than the mAs used in the pelvis due to the bony structures in the pelvis. The radiation dose given also depends on the patient's size with more dose required for larger patients and less for smaller patients. This is regulated according to the body thick- ness as illustrated in Table-2. Maximum mAs to be used can also be set in this Auto- $m A^{\mathrm{TM}}$ feature. Figure-1D illustrates the average dose generated during each scan and the total dose report.

\section{IMAGINGAND SURGICAL PLANNING}

Surgical planning is usually enhanced by the information obtained from imaging studies: extent of the tumor and its location in the kidney; proximity to

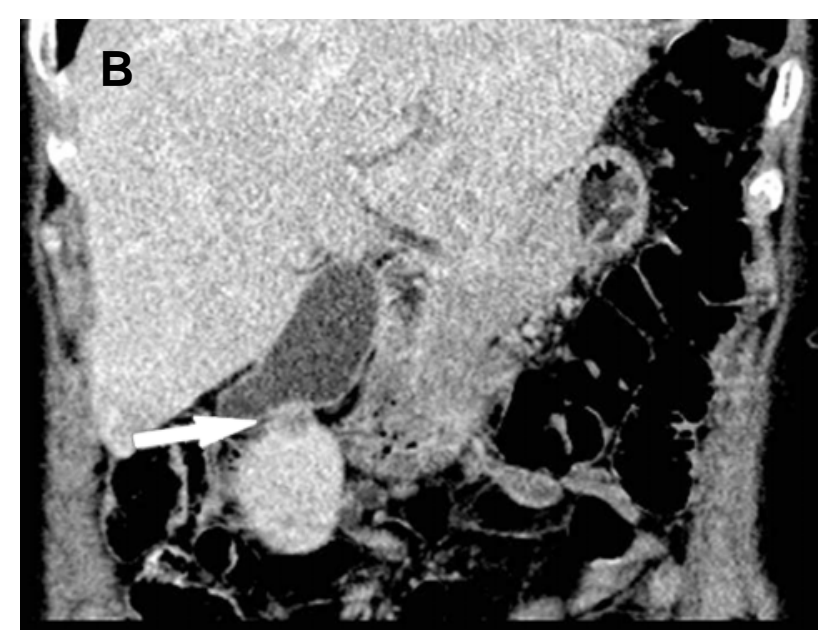

Figure 2 - The axial sections in this case failed to show the gallbladder involvement as clearly as the sagittal reformatted image did. A) Axial section through the abdomen showing the small renal cell carcinoma (white arrow) and the gallbladder adjacent to it (arrowhead). B) Coronal image showing involvement of the gallbladder seen as the loss of fat plane and thickening in the gallbladder wall (white arrow). The gallbladder had to be resected during surgery as it was adherent to the tumor and histopathology showed extension of the tumor into the gallbladder. 
Table 2 - The mAS is regulated according to the body thickness as per the table illustrated below.

\section{Patient Size}

(AP diameter from lateral scanogram)

\section{Auto $\mathbf{m A}^{\mathrm{TM}}$ Range}

$100-550$

$100-700$

$100-750$

$350-660$
Upper Limit of mAs

Measured width $<28 \mathrm{~cm}$

Measured width $29-34 \mathrm{~cm}$

Measured width $35-41 \mathrm{~cm}$

Measured width $>41 \mathrm{~cm}$
220

200

375

528 the renal collecting system and renal vessels; presence of fat planes between the tumor and other structures (for e.g., liver, colon and posterior abdominal wall muscles); tumor extension into the renal vein or IVC and the level of this extension. The presence of intrabdominal metastasis, the function of the

Table 3-Tumor.

Renal hilum

Close proximity to adjacent organs

Metastases

Size of mass

Location on kidney

Collateral vessels
Vessel anomalies, lymph nodes wrapped around vessels

Left side: pancreas, spleen, aorta/branches, iliacs, psoas, colon Right side: liver/gallbladder, IVC, colon, duodenum, iliacs

Liver, LN, adrenals, opposite kidney

Staging

Possible radiofrequency ablation (laparoscopic or percutaneous, partial nephrectomy

Upper, mid or lower

Anterior, posterior or lateral

Involvement of collecting system

Possible radiofrequency ablation (laparoscopic or percutaneous, partial nephrectomy

Arteries, veins

Table 4 - Inferior vena cava (IVC).

Level of thrombus

Possible invasion of IVC wall

Distension of IVC

Invasion of IVC branches and extent.
Infrahepatic, intrahepatic, hepatic veins, IVC above diaphragm, atrium.

Obstruction, extent of bland thrombus above and below IVC tumor thrombus 

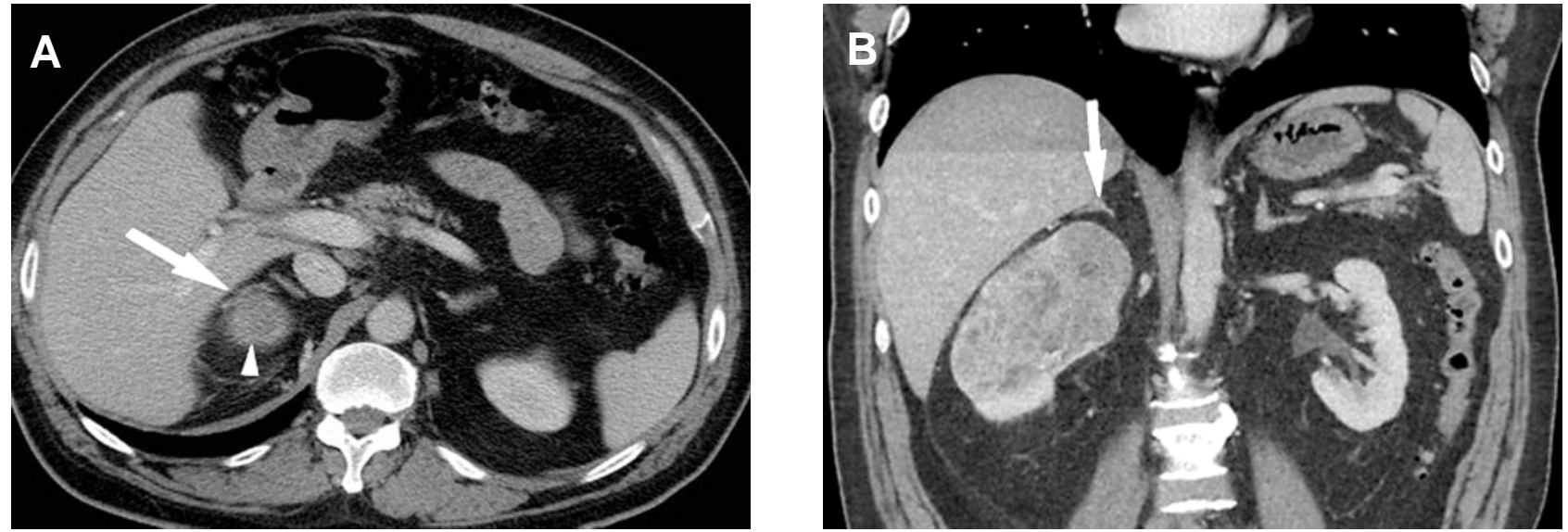

Figure 3-Noninvolvement of the adrenal shown more clearly on the coronal reformat. A) Axial image through the superior portion of the right kidney illustrating the tumor (arrowhead) in close proximity to the right adrenal gland (arrow). Fat plane between the tumor and the adrenal is not well seen. B) Note that the fat plane is much better seen on this coronal reformatted image confirming non-involvement of right adrenal gland.

contralateral kidney, and the appearance of the adrenal glands are also reliably assessed (12), Tables-3 and 4.

\section{CONCLUSION}

64 slice CT imaging provides an improvement in the preoperative analysis of renal masses. The reformatted images in multiple planes and cardiacgated sequences can offer additional help in surgical planning and reformatted images provide detailed information about arterial and venous collaterals and invasion of adjacent organs.

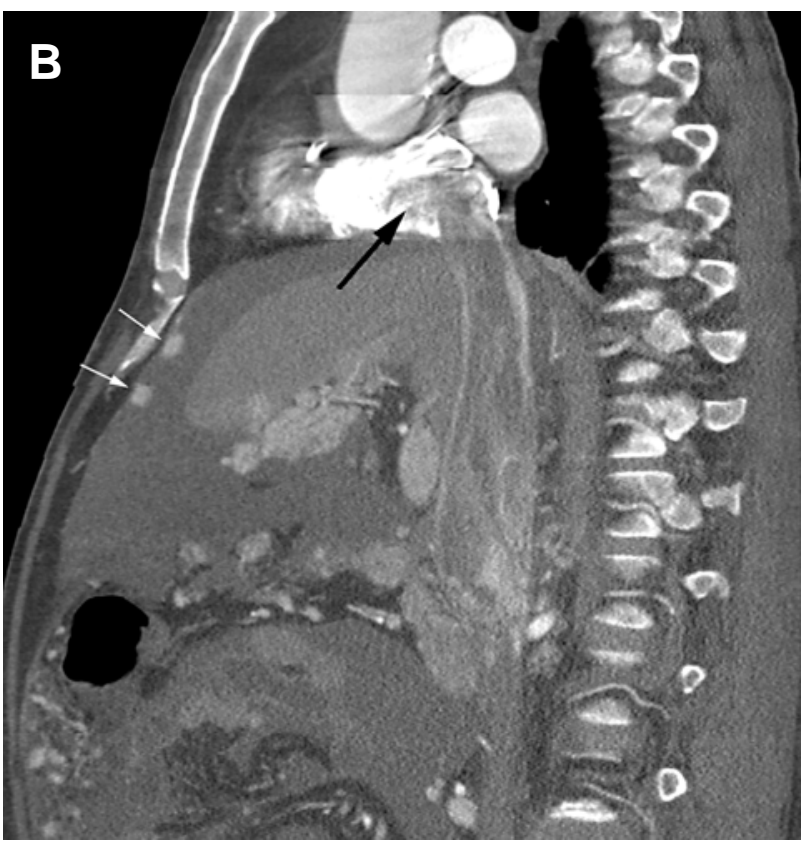

Figure 4-Tumor thrombus extent shown much better on cardiac-gated reformats. This study was performed by cardiac gating to assess the extent of tumor extension into the inferior vena cava. A) Axial image - tumor is seen to extend into the right atrium and into the right ventricle as well (black arrows). B) Sagittal reformat - note peritoneal metastasis (white arrow) and extend into the right atrium and into the right ventricle well (black arrow). 

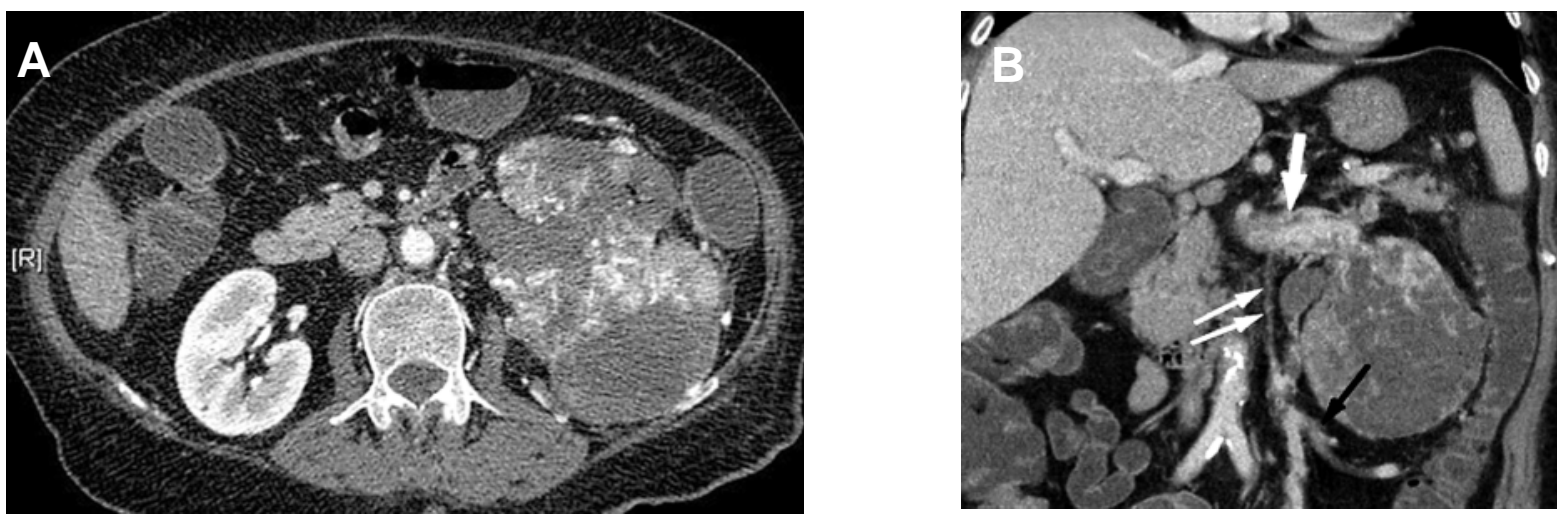

Figure 5-Ovarian vein thrombosis seen much better on the coronal reformat. A) Axial section demonstrates the large mass in the left kidney with multiple venous collaterals around it. B) Coronal reformatted image illustrates ovarian vein thrombosis (small double white arrows), which was missed on the axial section. Note the large thrombus in the left renal vein (large white arrow); the collaterals (black arrow) drain retrograde through the patent proximal ovarian vein.
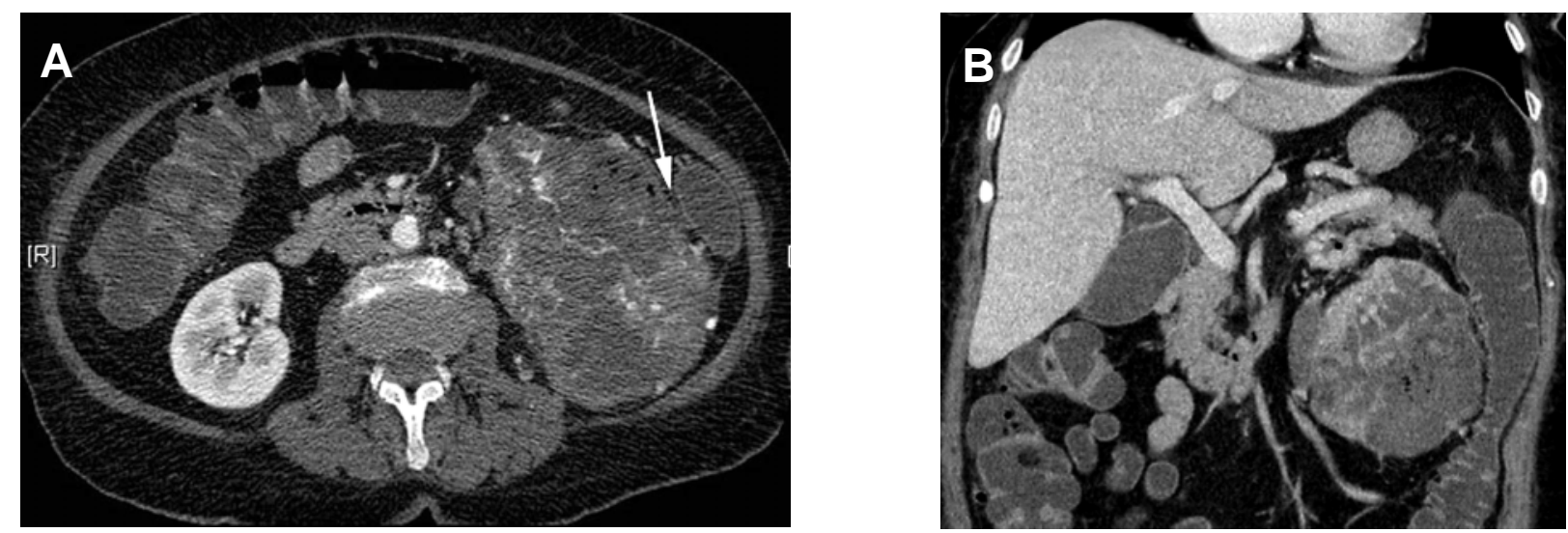

Figure 6-Relationship to the colon seen better on coronal reformat. A) Axial section through the left renal mass showing fat stranding (white arrow) suggesting extension of the mass into the colon. B) Coronal reformatted image illustrating the extent of contact of the mass with the colon but not confirming invasion. The colon was not involved at surgery.
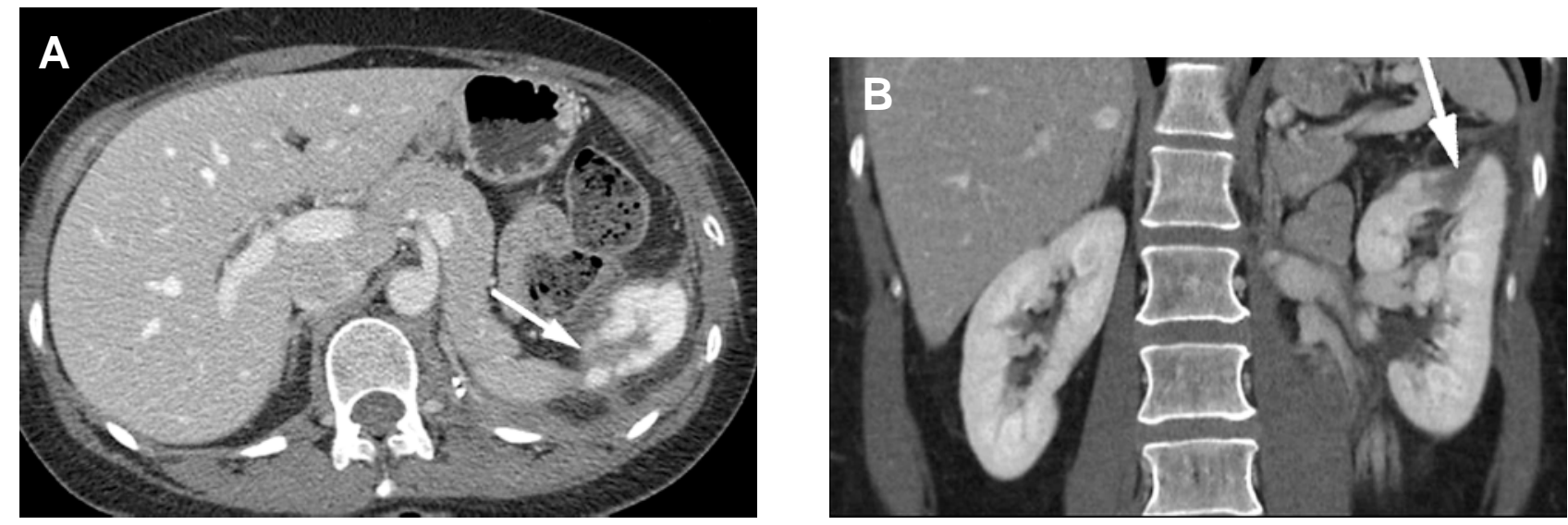

Figure 7- Postoperative CT showing scar tissue without recurrence of tumor. A) Axial section shows irregularly enhancing area in the upper pole of the left kidney (white arrow). B) Coronal reformats through the same area showed this to be only scar tissue (white arrow). 


\section{CONFLICT OF INTEREST}

None declared.

\section{REFERENCES}

1. Heidenreich A, Ravery V, European Society of Oncological Urology: Preoperative imaging in renal cell cancer. World J Urol. 2004; 22: 307-15.

2. Smith D: Renal Cancer. Urology. 2002; 20: 301-4.

3. Russo P: Renal cell carcinoma: presentation, staging, and surgical treatment. Semin Oncol. 2000; 27: 160-76.

4. Cohan RH, Sherman LS, Korobkin M, Bass JC, Francis IR: Renal masses: assessment of corticomedullaryphase and nephrographic-phase CT scans. Radiology. 1995; 196: 445-51.

5. Kopka L, Fischer U, Zoeller G, Schmidt C, Ringert RH, Grabbe E: Dual-phase helical CT of the kidney: value of the corticomedullary and nephrographic phase for evaluation of renal lesions and preoperative staging of renal cell carcinoma. AJR Am J Roentgenol. 1997; 169: 1573-8.

6. Szolar DH, Kammerhuber F, Altziebler S, Tillich M, Breinl E, Fotter R, et al.: Multiphasic helical CT of the kidney: increased conspicuity for detection and characterization of small $(<3-\mathrm{cm})$ renal masses. Radiology. 1997; 202: 211-7.

7. Yuh BI, Cohan RH: Different phases of renal enhancement: role in detecting and characterizing renal masses during helical CT. AJR Am J Roentgenol. 1999; 173: 747-55.

8. Israel GM, Bosniak MA: How I do it: evaluating renal masses. Radiology. 2005; 236: 441-50.

9. Saunders HS, Dyer RB, Shifrin RY, Scharling ES, Bechtold RE, Zagoria RJ: The CT nephrogram: implications for evaluation of urinary tract disease. Radiographics. 1995; 15: 1069-85.

10. Birnbaum BA, Jacobs JE, Ramchandani P: Multiphasic renal CT: comparison of renal mass enhancement during the corticomedullary and nephrographic phases. Radiology. 1996; 200: 753-8.

11. Macari M, Bosniak MA: Delayed CT to evaluate renal masses incidentally discovered at contrast-enhanced CT: demonstration of vascularity with deenhancement. Radiology. 1999; 213: 674-80.

12. Kaplan S, Ekici S, Dogan R, Demircin M, Ozen H, Pasaoglu I: Surgical management of renal cell carcinoma with inferior vena cava tumor thrombus. Am J Surg. 2002; 183: 292-9.

\author{
Correspondence address: \\ Dr. Manjiri Dighe \\ Assistant Professor \\ Department of Radiology, Box 357115 \\ University of Washington Medical Center, \\ Seattle, WA, 98195, USA \\ Fax: + $1206598-0252$ \\ E-mail:dighe@u.washington.edu
}

